\title{
Examination of Inactive Time \& Physical Activity in College-Aged Students
}

Katelyn Snyder, Beth Larouere. Slippery Rock University, Slippery Rock, PA

It is widely accepted that regular exercise and physical activity (PA) reduce the risk of many chronic diseases. Not participating in PA is reported to be the biggest public health concern of the 21 st century. New evidence suggests that time spent in sedentary behaviors presents an independent cardiovascular and metabolic disease risk. PURPOSE: To examine time spent in PA and inactivity as well as to compare accelerometry to self-reported inactive time. METHODS: Subjects $(n=49)$ reported to the laboratory on 2 separate occasions. At the first visit, participants completed the Paffenbarger questionnaire (PAFF) to assess baseline activity and sedentary patterns. Also, the activPAL3 accelerometer was placed on the subjects' thigh and worn continuously for 7 consecutive days to obtain objective measures of PA and sedentary behavior. The subjects returned to the lab 8 days later to return the accelerometer and complete the PAFF questionnaire again for the timeframe the activPAL3 was worn. RESULTS: Accelerometry revealed that mean time spent (hrs/day) was: $18.58( \pm 1.2)$ being inactive, 3.43( \pm .89$)$ standing, and 1.99 $(+.52)$ stepping. A strong inverse relationship $(\mathrm{r}=-.726)$ exists between mean inactivity and mean steps/day. Those who met the 10,000 steps/day recommendation $(55.1 \%)$ had significantly less time spent in inactivity $(p<.001)$ than those who did not. Significantly more time $(p<.01)$ was found between the number of steps taken during the week as compared to the weekend. However, there was no significant difference between mean inactive time during the week as compared to the weekend. Participants self-reported significantly less time spent in inactivity than accelerometer recorded ( $\mathrm{p}<.01)$. CONCLUSION: Even when the 10,000 step/day recommendation was met, a large proportion of the day (75\%) was spent in sedentary behaviors. Interventions to decrease time spent in prolonged inactivity may be warranted in addition to increasing PA.

Supported by a Slippery Rock University College of Health, Environment \& Science grant. 